INT. J. REMOTE SENSING, 1999, VOL. 20, NO. 17, 3247-3263

\title{
Radiometric characterization of the ROCSAT Ocean Color Imager
}

\author{
WEI-SONG LIN†, JENN-YII WU, HENG-JE CHIU, \\ CHUN-SHENG CHEN and YU-JEN CHANG
}

Institute of Electrical Engineering, National Taiwan University, Taiwan, Republic of China

\section{(Received 8 October 1998)}

\begin{abstract}
The Ocean Color Imager (OCI), a multispectral optical imager for observing pigment distributions in low-latitude oceans, has been successfully built and parameterized. To recover at-sensor radiance data, a mathematical model was developed to characterize its radiometric response; the corresponding parameters were identified initially by pre-flight calibration and were adapted in-flight by a method of cross-platform calibration. The cross-platform in-flight calibration was carried out to compare radiance data with data measured by the vicarious orbital sensor at the cross points to determine possible changes in the parameters and performance of the OCI. The radiometric model, the pre-flight parameters and the method of cross-platform in-flight calibration are reported here.
\end{abstract}

\section{Introduction}

The Ocean Color Imager (OCI), one of the payloads of the ROCSAT-1 satellite launched on January 27 1999, is a product of the National Space Program Office (NSPO) of the National Science Council (NSC) of Taiwan built by the NEC Corporation of Japan. Technically, OCI is an all-refractive, pushbroom and nadirviewing imaging sensor designed to investigate pigment distributions in low-latitude oceans $\left( \pm 35^{\circ}\right)$ by measuring six spectral bands in the visible and near-infrared (NIR) spectrum. Pigment distribution data on a large scale are expected to contribute to the understanding of ocean dynamics, chlorophyll variation and oceanic primary production. The information-containing radiance backscattered out of the water and transmitted to the top of the atmosphere is only a small portion of the radiance that is measured by a space-borne ocean color sensor (Gordon 1998). To determine the water-leaving radiance, OCI data require several levels of radiometric correction to remove the effects of disturbances from inside the instrument itself, scattering from the atmosphere and direct reflectance from the ocean surface (Schowengerdt 1997a). Corrections for instrument disturbance require a radiometric model of the OCI and the corresponding parameters calibrated in both pre- and in-flight stages (Rao and Chen 1994). The pre-flight calibration and validation activities of the OCI were carried out at the NEC Corporation in Yokohama, Japan at instrument level

$\dagger$ Department of Electrical Engineering, National Taiwan University, No. 1, Sec. 4, Roosevelt Rd., Taipei, Taiwan, Republic of China. e-mail address: weisong@cc.ee.ntu.edu.tw 
and at the NSPO in Taiwan for integration with the ROCSAT-1 satellite. Using design and testing data, this paper reports on the radiometric model, the pre-flight parameters and the method of cross-platform in-flight calibration. Variations in the offset and degradation of the performance of the optics with time in orbit have been considered. The results have been implemented into computer software to convert the OCI output into at-sensor radiance data. The aims of this paper are to characterize the radiometric response of the OCI by modelling and calibration and to summarize its major characteristics.

\section{Characteristics of $\mathrm{OCI}$}

Figures $1(a)$ and $(b)$ show schematic diagrams of the OCI and its optics (Narimatsu et al. 1997 a). The instrument is basically an electro-optical assembly with four independent telecentric dioptric systems to accommodate seven spectral bands (these seven bands are actually six plus a back-up band). The band selections consider the spectral characteristics of in-water optical constituents, spectral transmittance of the atmospheric constituents and data fusion with other space-borne ocean colour sensors. As shown in table 1, the six bands are specified to centre at $443,490,510,555,670$ and $865 \mathrm{~nm}$. The back-up band is centred at $555 \mathrm{~nm}$ and is denoted as Band 7. The desired bandwidth for Bands $1-5$ and 7 is $20 \mathrm{~nm}$ and for Band 6, $40 \mathrm{~nm}$.

The physical implementation of the OCI is subject to the deployment of total mass, envelope and other resources on the ROCSAT-1 satellite. To meet the radiometric specifications without violating the deployment, Bands 1 and 3 are implemented to draw radiance from one single telescope, as shown in figure 1(b). A lens system and a phase plate focus and depolarize the light beam respectively, and a prism beam splitter distributes the incoming radiance to the two linear charge-coupled device (CCD) arrays assembled on each of the two focal planes. The interference filter in front of each CCD array determines the centre wavelength and bandwidth of the corresponding band. Bands 2 and 4, and Bands 5 and 6 are also coupled and used in the same way. Band 7, which is a back-up of Band 4, uses a stand-alone telescope with the same structure. The four sets of optics and seven detector assemblies were manufactured and aligned accurately to obtain co-registration errors no larger than 0.65 instantaneous field-of-view (IFOV) along-track and 0.97 IFOV across-track. Construction and testing of the OCI flight model has been completed; its major parameters and characteristics are summarized in table 2 (Narimatsu et al. 1997b).

A TH7811 (Thomson-CSF) CCD array was chosen as the detector for each band. This component has 1728 cells arranged as a linear array and operates the particular function of anti-blooming control. The photosensitive area of each cell is approximately $13 \mu \mathrm{m} \times 13 \mu \mathrm{m}$. In OCI pushbroom scanning, the cells of each band are organized as shown in figure 2 to give a total of 896 pixels with 832 double-cell pixels (dp) separated equally on both sides and 64 single-cell pixels (sp) at the central part. A double-cell pixel with a $115.8 \mathrm{~ms}$ integration time interval is specified to have an approximately $800 \mathrm{~m} \times 800 \mathrm{~m}$ footprint at nadir in a $600 \mathrm{~km}$ orbit. In the same situation, a single-cell pixel will have a footprint of only $400 \mathrm{~m} \times 800 \mathrm{~m}$. All the 896 pixels combined with the wide FOV optics and the motion of the satellite are expected to scan with a swath width no less than $690 \mathrm{~km}$ in a $600 \mathrm{~km}$ orbit. Table 2 lists the implemented swath values. The OCI achieves a high performance in the across-track modulation transfer function (MTF), but this function in Band 6 is 


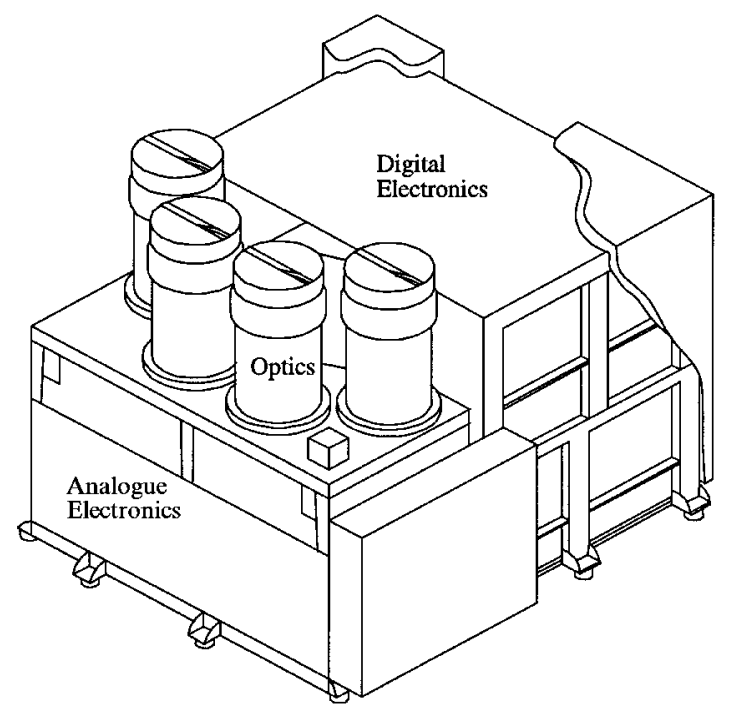

(a)

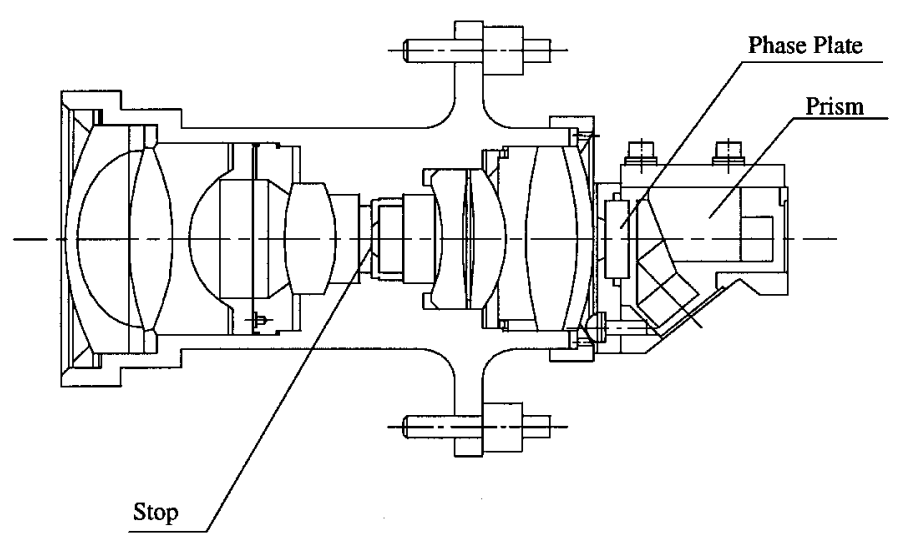

(b)

Figure 1. A schematic view of OCI: (a) an overview; (b) the telecentric dioptric optics.

exceptionally low due to its larger bandwidth. The high signal-to-noise ratio (SNR), obtained mainly because of the very low noise electronic implementation, combined with 12-bit digitization ensures the accuracy of OCI data.

\section{Approximate radiometric model of OCI}

The OCI is an electro-optical sensor that converts at-sensor radiance into appropriate digital counts. According to the design and physical implementation, its signal flow can be represented as shown in figure 3. The at-sensor radiance is transformed 
Table 1. Bands of the OCI.

\begin{tabular}{lccl}
\hline Band & $\bar{\lambda}(\mathrm{nm})$ & $\Delta \lambda(\mathrm{nm})$ & \multicolumn{1}{c}{ Phenomenon } \\
\hline 1 & 443 & 20 & Chlorophyll absorption (blue) \\
2 & 490 & 20 & Pigment \\
3 & 510 & 20 & Chlorophyll absorption (green) \\
$4 / 7$ & 555 & 20 & Hinge point, sediments (yellow) \\
5 & 670 & 20 & Aerosol correction (red) \\
6 & 865 & 40 & Aerosol correction (NIR) \\
\hline
\end{tabular}

sequentially by the telescope, detector, analogue electronics, multiplexer, gain selector and analogue to digital converter (A/D) to obtain digital counts. The signals picked up by the detectors of the seven bands are sequentially multiplexed to a gain selector and an analogue to digital converter. The gain selector provides normal (1.0), low (0.5) and high (2.0 for Bands 1-6, 6.0 for Band 7) gains in compliance with the signal level. To investigate the radiometric response of a single spectral band, the key components affecting the performance were identified (figure 4). The boxes with heavy rules (gain selector and A/D) represent the common units of the seven bands. The major factors that affect the radiometric or instrument response of the OCI exist mainly in components such as the optics, the detector and the electronics. In the optics, these factors are characterized by the transmission of the lens, MTF, FOV, stray light of the optical assembly, depolarization of the phase plate, and centre wavelength $(\bar{\lambda})$ and bandwidth $(\Delta \lambda)$ of the interference filters. The responsivity and dark signal of the CCD are the most important factors to be considered in the optical to electronic conversion. The analogue and digital electronics transform the electronic signal into appropriate digital counts with the response function characterized by the amplifier or buffer gain and bias, digitization and noise. Since these factors characterize the response functions of the optics, the detector, the electronics, as well as the whole OCI instrument, they were specified in the OCI requirements in detail. The manufacturer satisfied these requirements with appropriate materials, components, construction and testing procedures (NSPO 1993). The engineering phase of the OCI was obviously complicated and difficult.

The overall instrument response function of the $\mathrm{OCI}$ is theoretically the convolution of the component response functions (Schowengerdt 1997b). However, in order to simplify data conversion and parameter calibration, it is assumed that, for a particular integration time interval, the spectral response of the OCI is an average constant over an effective spectral band, and the spatial response is an average constant over the effective area of a detector element. The band-, space- and timeintegrated at-sensor radiance measured by the OCI can then be represented by the following equation:

$$
L_{b p}(t)=\iiint_{\Delta \tau \Delta^{A}{ }_{\Delta \lambda}} L(\lambda, A, \tau) \mathrm{d} \lambda \mathrm{d} A \mathrm{~d} \tau
$$

where $L_{b p}$ denotes the measured radiance at a particular CCD pixel $p$ in band $b,(t)$ is used to denote the time of measurement (similarly $(\lambda, A, \tau)$ ), $\Delta \lambda$ is the effective spectral band, $\Delta A$ is the effective area of an CCD pixel and $\Delta \tau$ is the integration time interval. Then referring to figure 4 , the radiometric response of the OCI can be 
Table 2. Major parameters and characteristics of the OCI.

\section{Instrument characteristics}

Imaging method

Optics

Dimensions (mm)

Mass $(\mathrm{kg})$

Power consumption (W)

Design life

Reliability

Orbit

Operating modes

Gain selection

Image data rate (limit)

Detectors

Pixels per band

Integration time interval (ms)

Signal digitization

Absolute radiance accuracy

Spectral co-registration error

(with respect to Band 4)

Polarization sensitivity

Anti-blooming

\section{Band characteristics}

Band

Swath width $(\mathrm{km})$ $(h=600 \mathrm{~km})$

FOV

GIFOV (m)

(Ground IFOV) ( $h=600 \mathrm{~km}$ at nadir)

IFOV (at nadir)

Centre wavelength

(nm) (specification)

Bandwidth (nm)

(specification)

MTF (across track) (pixel no. 416)

MTF (along track) (pixel no. 416)

SNR (pixel no. 416)

Mean radiance

Saturation radiance

Out of band spectral rejection $(\%)$
Pushbroom scanning

Four telecentric dioptric systems (lens system)

$378.8(X) \times 348.6(Y) \times 342.0(Z)$

\section{2}

Peak, 33; standby, 17.4

2 years in orbit

0.991 .7 bands operating at the end of 2 years in orbit (3\% duty cycle)

Incline $35^{\circ}$; altitude $600 \mathrm{~km}$

period $96.7 \mathrm{~min}$; speed $7.56 \mathrm{~km} \mathrm{~s}^{-1}$

FB(1-7), NI-A(1-6), NI-B(1-3, 5-7), RGB(1,3,5), $\mathrm{CA}(4,7)$

Normal $=1.0$, low $=0.5$, high $=2.0(6.0$ for Band 7$)$ $<654.8 \mathrm{kbps}(655.5 \mathrm{kbps})$

Linear CCD arrays, 1728 cells, $13 \mu \mathrm{m} \times 13 \mu \mathrm{m}$

896 pixels $(832$ double +64 single cells $)$

115.8

12 bits per pixel

$5 \%$ or better at BOL (Begin-of-Line)

along track: $\leqslant 0.65$ IFOV

across track: $\leqslant 0.97$ IFOV

$\leqslant 2 \%$

$\leqslant 10 \%$ of $V_{\text {sat }}$ at the $10^{\text {th }}$ pixel $\left(E=6 \times E_{\text {sat }}\right)$

$\begin{array}{ccccccc}\text { B1 } & \text { B2 } & \text { B3 } & \text { B4 } & \text { B5 } & \text { B6 } & \text { B7 } \\ 701.6 & 702.2 & 702.2 & 702.4 & 702.0 & 702.3 & 702.1 \\ & & & & & & \\ 60^{\circ} 37^{\prime} & 60^{\circ} 40^{\prime} & 60^{\circ} 40^{\prime} & 60^{\circ} 40^{\prime} & 60^{\circ} 39^{\prime} & 60^{\circ} 40^{\prime} & 60^{\circ} 39^{\prime} \\ 42.3^{\prime \prime} & 11.1^{\prime \prime} & 5.3^{\prime \prime} & 57.0^{\prime \prime} & 26.4^{\prime} & 36.6^{\prime \prime} & 57.0^{\prime \prime} \\ 807.5 & 808.4 & 806.3 & 807.5 & 808.1 & 806.6 & 806.9\end{array}$

$\begin{array}{rrrcccc}4^{\prime} 37.6^{\prime \prime} & 4^{\prime} 37.9^{\prime \prime} & 4{ }^{\prime} 37.2^{\prime \prime} & 4^{\prime} 37.6^{\prime \prime} & 4{ }^{\prime} 37.8^{\prime} & 4{ }^{\prime} 37.3^{\prime \prime} & 4^{\prime} 37.4^{\prime \prime} \\ 444.0 & 491.6 & 511.9 & 554.6 & 670.0 & 868.9 & 554.7 \\ (443) & (490) & (510) & (555) & (670) & (865) & (555) \\ 20.0 & 20.1 & 19.6 & 18.5 & 18.5 & 40.3 & 18.5 \\ (20.0) & (20.0) & (20.0) & (20.0) & (20.0) & (40.0) & (20.0) \\ 0.77 & 0.78 & 0.78 & 0.76 & 0.66 & 0.53 & 0.79 \\ & & & & & & \\ 0.56 & 0.60 & 0.56 & 0.57 & 0.58 & 0.55 & 0.58\end{array}$

$\begin{array}{rrrrrrc}899.9 & 934.4 & 914.9 & 790.4 & 891.1 & 799.3 & 845.1 \\ 84.1 & 65.6 & 56.4 & 45.7 & 24.6 & 10.9 & 45.7 \\ 132.5 & 105.0 & 90.8 & 74.4 & 42.0 & 21.3 & 74.4 \\ 0.10 & 0.22 & 0.09 & 0.11 & 0.19 & 0.07 & 0.09\end{array}$

approximated mathematically by a linear, slowly time-varying system as follows:

$$
C_{b p g}(t)=g F^{\mathrm{e}}(t)\left[F_{b p}^{\mathrm{o}}(t) L_{b p}(t)+D_{b p}^{\mathrm{o}}(t)\right]+D^{\mathrm{e}}(t)
$$

where $C, F$ and $D$ denote the digital counts, sensor gain and offset respectively; $g$ is the selected gain; subscripts denote a particular band $b$, pixel $p$ and selected gain $g$; and superscripts $o$ and e represent the roughly optical-related and common electronic 
$691.2 \mathrm{~km}$

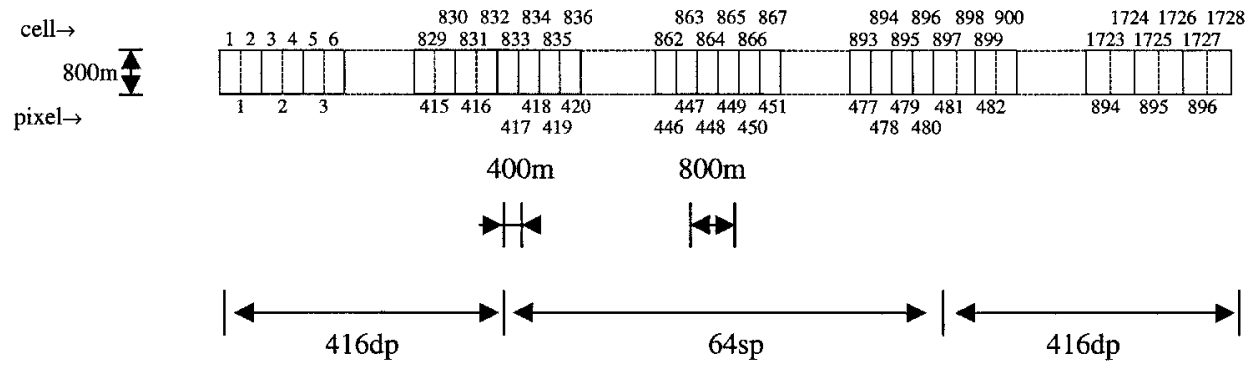

Figure 2. Organization of CCD cells.

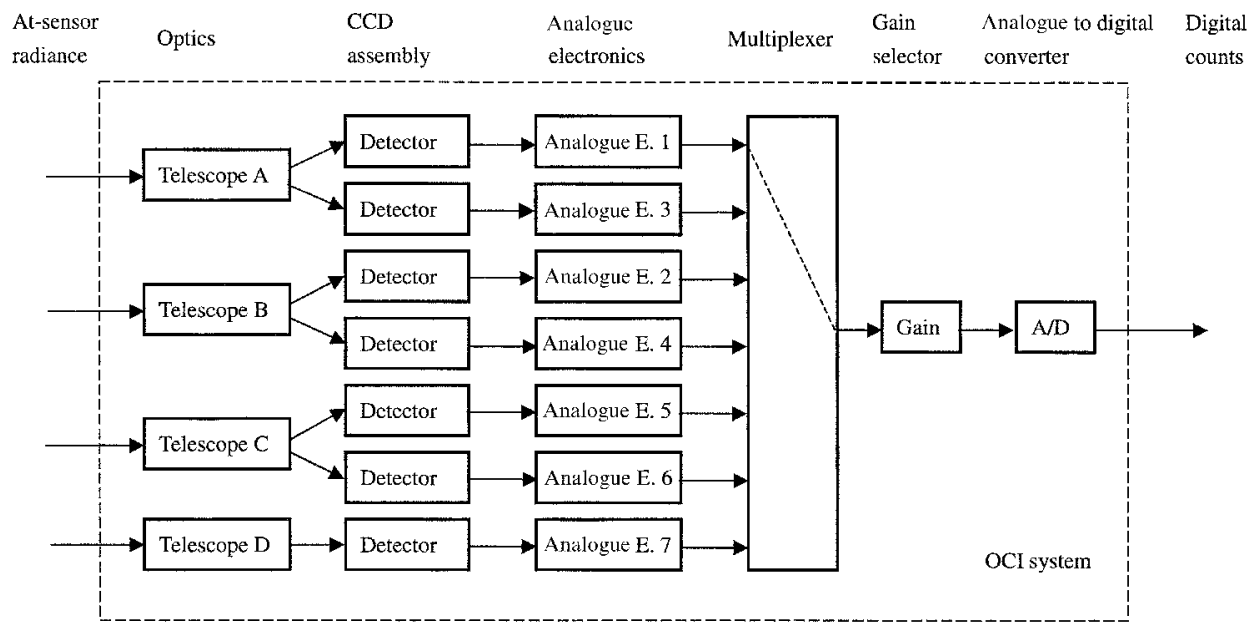

Figure 3. Signal flow and main components of the $\mathrm{OCI}$, where each numeric number denotes the corresponding band.

portions of the OCI. The terms on the right-hand side of equation (2) can be rearranged as follows:

$$
C_{b p g}(t)=g F_{b p}(t) L_{b p}(t)+D_{b p g}(t)
$$

where

$$
F_{b p}(t)=F^{\mathrm{e}}(t) F_{b p}^{\mathrm{o}}(t)
$$

and

$$
D_{b p g}(t)=g F^{\mathrm{e}}(t) D_{b p}^{o}(t)+D^{\mathrm{e}}(t)
$$

For the convenience of calibration, equation (3) can be rewritten by referring to the pre-flight sensor gain as follows:

$$
C_{b p g}(t)=\alpha_{b p}(t) g F_{b p}\left(t_{0}\right) L_{b p}(t)+D_{b p g}(t)
$$




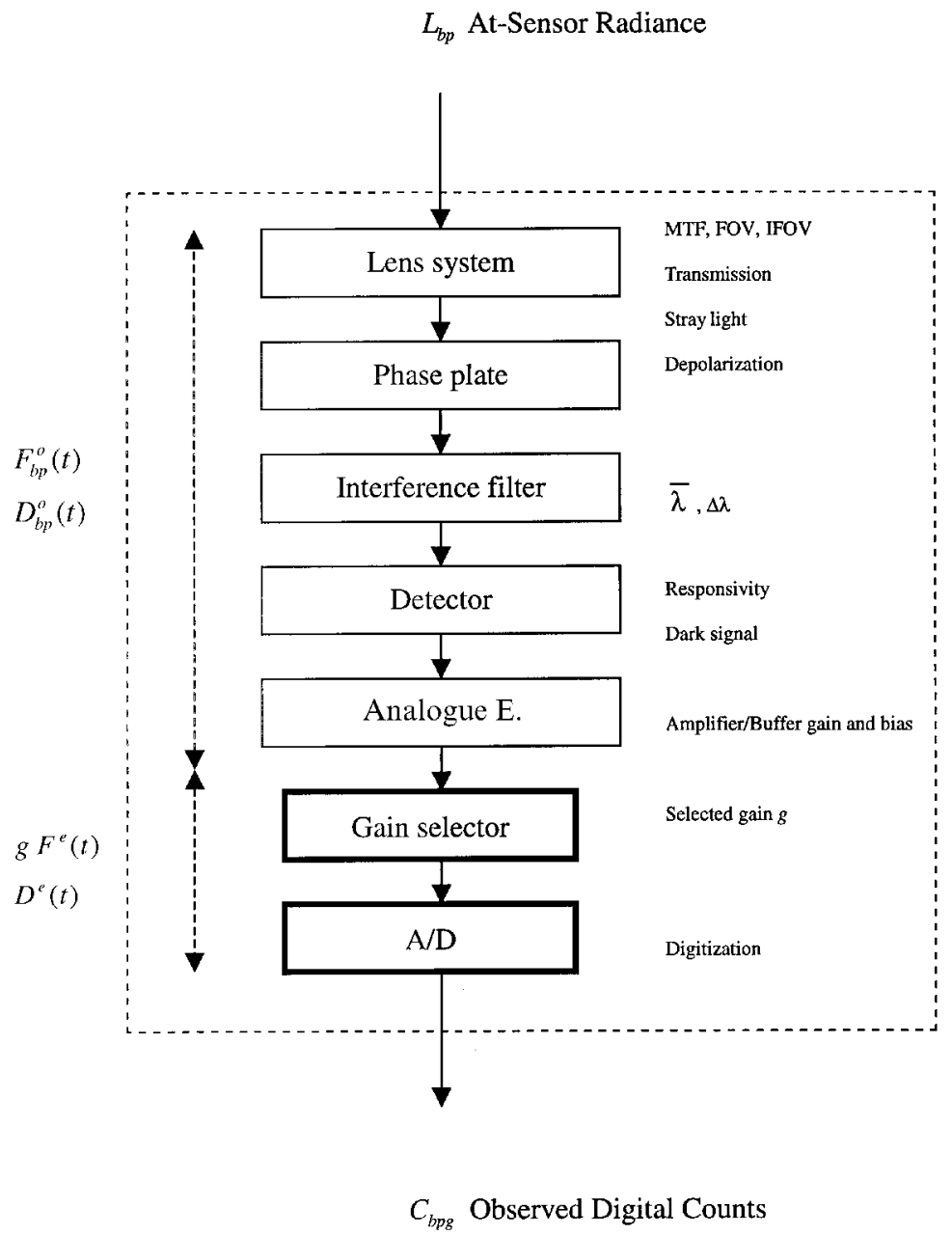

Figure 4. Major factors affecting the performance of a single band.

where $t_{0}$ denotes the time of pre-flight calibration and

$$
\alpha_{b p}(t)=\frac{F_{b p}(t)}{F_{b p}\left(t_{0}\right)}
$$

is designated as the relative sensor gain. Equations (6) and (7) form an approximate radiometric model for parameter calibration and data conversion of the OCI. Using this model, the pre-flight calibration needs to identify $F_{b p}\left(t_{0}\right)$ and $D_{b p g}\left(t_{0}\right)$ and the in-flight calibration is requested to estimate $\alpha_{b p}(t)$ and $D_{b p g}(t)$ periodically or frequently during the lifetime of the OCI.

\section{Pre-flight parameters of OCI}

In the pre-flight calibration

$$
\alpha_{b p}\left(t_{0}\right)=1.0
$$


and the approximate radiometric model becomes

$$
C_{b p g}(t)=g F_{b p}\left(t_{0}\right) L_{b p}(t)+D_{b p g}\left(t_{0}\right)
$$

where $g=1.0$ is a convenient choice. Pre-flight parameters of the OCI at room temperature were obtained in October 1997. The primary standard of radiance was a copper point blackbody source at $1084.62^{\circ} \mathrm{C}$. The secondary standard of radiance was a variable-temperature transfer blackbody (VTTB) operated between $800^{\circ} \mathrm{C}$ and $1500^{\circ} \mathrm{C}$. The specifications of the $\mathrm{OCI}$ integrating sphere are shown in table 3 . The sphere was calibrated in November 1996 relative to the primary standard using the VTTB and a double grating monochromator. The procedure has been described fully by Narimatsu et al. (1997c); it is summarized briefly here as follows. The radiance of the primary standard was calculated using Planck's law. A monochromator was used to compare the spectral radiance of the VTTB with the primary standard. Then the VTTB was transported to the working place and applied to validate the radiance of the sphere. For Bands 1-4 and Band 7, the VTTB was set to $1372^{\circ} \mathrm{C}$; for Bands 5 and 6 the temperature was at $957^{\circ} \mathrm{C}$.

In the calibration the OCI was kept at room temperature, set to normal gain and calibrated by observing a variety of sphere output radiances. The output radiance of the sphere was selected to test eight points with magnitudes less than the saturation radiance of each band. Recording the digital counts obtained by the OCI corresponding to the applied radiance and applying linear regression can approximate the parameters in equation (9). The values of $F_{b p}\left(t_{0}\right)$ and $D_{b p g}\left(t_{0}\right)$ at room temperature are available in the literature (Narimatsu et al. 1997c). Figure 5 depicts the whole set of $F_{b p}\left(t_{0}\right)$. The difference between single- and double-cell pixels results in the low sensor gains in the central portions of the curves. Since the CCD is temperature sensitive, when the OCI is in normal observation mode, the temperature of each CCD detector will be controlled to within $10 \pm 3^{\circ} \mathrm{C}$. Therefore, in spite of the fact that the pre-flight sensor gain is measured at room temperature only, the offset was also measured for the CCD being maintained at around $10^{\circ} \mathrm{C}$. The complete set of pre-flight offsets was obtained for each band, pixel and selected gain by the NSPO in July 1998 after the OCI was integrated with the ROCSAT-1 satellite. Table 4 lists the mean values and standard deviations of the pre-flight offsets.

\section{Parameter adaptation by in-flight cross-calibration}

Although the OCI was designed without moving components in order to achieve high reliability, as indicated in table 2, one major cause of variability in the

Table 3. Specifications of the OCI integrating sphere source.

\begin{tabular}{|c|c|c|c|c|c|c|c|}
\hline Inner diameter $(\mathrm{m})$ & \multicolumn{7}{|l|}{2} \\
\hline Aperture diameter $(\mathrm{mm})$ & \multicolumn{7}{|l|}{500} \\
\hline Coating on inner wall & \multicolumn{7}{|c|}{ Barium sulphate } \\
\hline Lamp configuration & \multicolumn{7}{|c|}{26 lamps, $0-100 \mathrm{~V} \mathrm{dc} \pm 0.01 \%$} \\
\hline $\begin{array}{l}\text { Monitor detector } \\
\text { Cooling }\end{array}$ & \multicolumn{7}{|c|}{ Silicon photodiode with infrared blocking filter } \\
\hline Band & 1 & 2 & 3 & 4 & 5 & 6 & 7 \\
\hline No. of lamps turned on & 26 & 26 & 26 & 26 & 13 & 13 & 26 \\
\hline Lamp voltage $(\mathrm{V})$ & 90.0 & 77.0 & 70.0 & 58.0 & 46.0 & 25.8 & 58.0 \\
\hline $\begin{array}{l}\text { Temp. of cal. blackbody } \\
\left({ }^{\circ} \mathrm{C}\right)\end{array}$ & 1372 & 1372 & 1372 & 1372 & 957 & 957 & 1372 \\
\hline
\end{tabular}



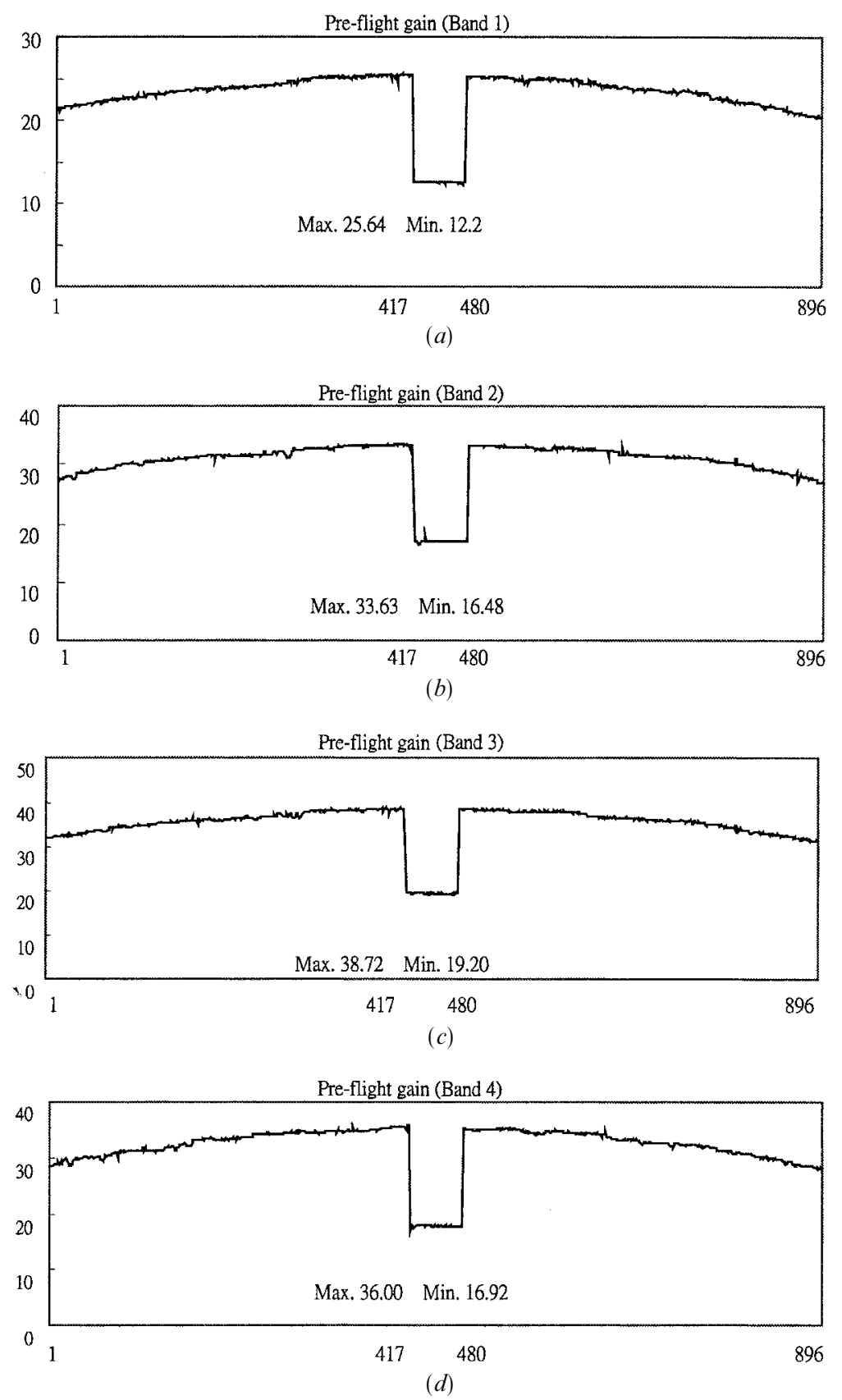

radiometric response is optical transmittance degradation as a result of exposure to radiation and contamination of the optics by outgassing. Another possibility is mechanical shift of the optical assembly during launch. The continuity of pre-flight calibration data with in-flight data and long-term variations in performance should therefore be monitored. Using the radiometric model of equation (6), if at-sensor 


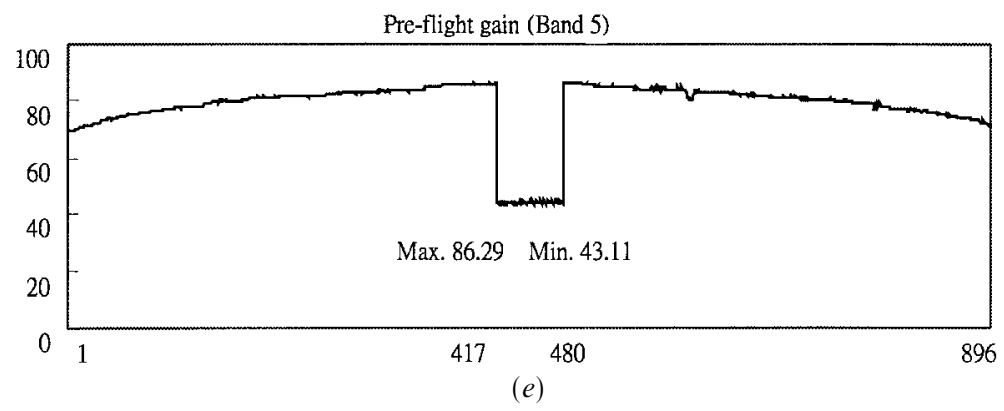

Pre-flight gain (Band 6)

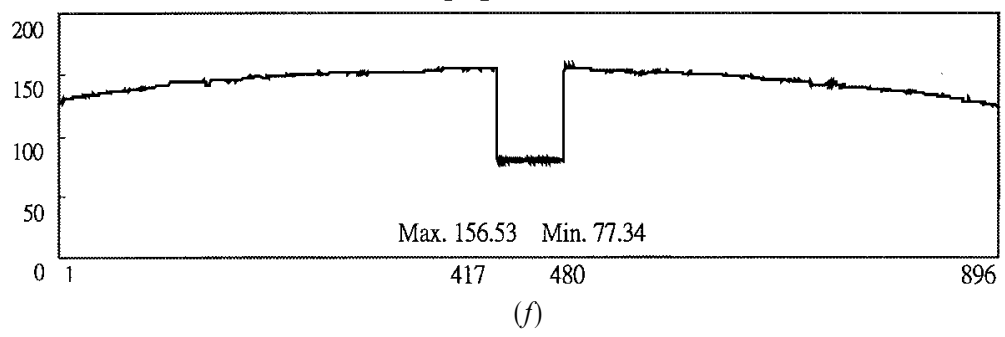

Pre-flight gain (Band 7)

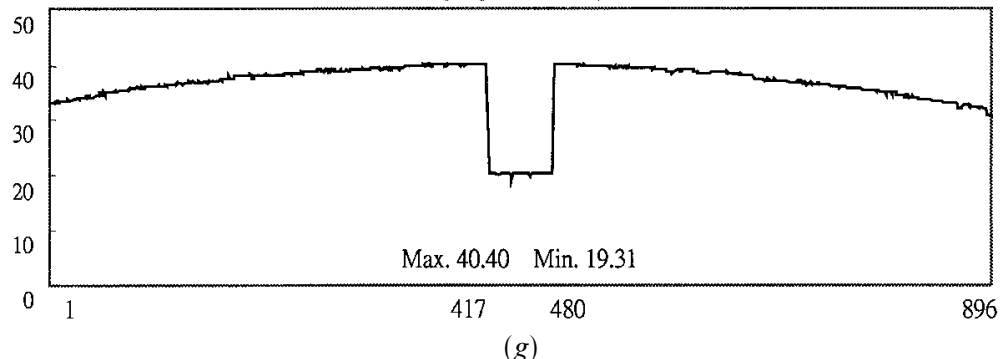

Figure 5. Plots of pre-flight sensor gain against pixel number. (a) Band 1, (b) Band 2, (c) Band 3, (d) Band 4, (e) Band 5, $(f)$ Band 6 and $(g)$ Band 7.

radiance data are available, the in-flight relative sensor gain can be assessed using

$$
\alpha_{b p}(t)=\frac{\hat{C}_{b p g}(t)-\hat{D}_{b p g}(t)}{g F_{b p}\left(t_{0}\right) \hat{L}_{b p}^{v}(t)}
$$

where ' ${ }^{\wedge}$, denotes the measured value, $\hat{C}_{b p g}(t)$ is the digital count obtained by the OCI, $\hat{D}_{b p g}(t)$ is the measured offset and $\hat{L}_{b p}^{v}(t)$ represents the known at-sensor radiance data. Since the offset can be estimated by observing the night side of the Earth, it remains to select an appropriate way or multiple ways to provide the at-sensor radiance data. For this purpose, on-board calibration using a lamp light source, blackbody radiator, moon light or diffused sunlight (Ono et al. 1996), and many vicarious and cross-calibration methods have been considered for orbital sensors (Slater et al. 1987, Gordon 1998, Che et al. 1991). Among these various methods, assessing the at-sensor radiance by vicarious measurements of the reflectance or radiance of some target site has been used with great success. Here, since several ocean colour sensors are expected to operate simultaneously during the mission time of the OCI (Salomonson et al. 1989, Hooker et al. 1992), apart from 
Table 4. Means and standard deviations of the pre-flight offsets.

\begin{tabular}{|c|c|c|c|c|c|c|}
\hline \multirow[b]{2}{*}{ Band } & \multirow[b]{2}{*}{$\begin{array}{c}\text { Gain } \\
\text { selection }\end{array}$} & \multirow[b]{2}{*}{$\begin{array}{c}\text { CCD } \\
{ }^{\circ} \mathrm{C}\end{array}$} & \multicolumn{2}{|c|}{ Double-cell pixels } & \multicolumn{2}{|c|}{ Single-cell pixels } \\
\hline & & & $\begin{array}{l}\text { Mean } \\
\text { offset }\end{array}$ & $\begin{array}{l}\text { Standard } \\
\text { deviation }\end{array}$ & $\begin{array}{l}\text { Mean } \\
\text { offset }\end{array}$ & $\begin{array}{l}\text { Standard } \\
\text { deviation }\end{array}$ \\
\hline 1 & 1.0 & 10.0 & 47.981 & 0.291 & 42.198 & 0.332 \\
\hline 2 & 1.0 & 10.3 & 47.981 & 0.291 & 42.198 & 0.332 \\
\hline 3 & 1.0 & 10.3 & 47.528 & 0.347 & 42.128 & 0.246 \\
\hline 4 & 1.0 & 10.3 & 48.190 & 0.324 & 42.316 & 0.224 \\
\hline 5 & 1.0 & 10.1 & 47.010 & 0.216 & 39.656 & 0.298 \\
\hline 6 & 1.0 & 9.7 & 46.804 & 0.181 & 41.488 & 0.223 \\
\hline 7 & 1.0 & 10.8 & 45.298 & 0.248 & 39.308 & 0.271 \\
\hline 1 & 2.0 & 9.7 & 98.422 & 0.417 & 85.446 & 0.650 \\
\hline 2 & 2.0 & 8.3 & 97.183 & 0.439 & 85.818 & 0.282 \\
\hline 3 & 2.0 & 10.2 & 99.693 & 0.553 & 87.136 & 0.458 \\
\hline 4 & 2.0 & 8.4 & 97.510 & 0.385 & 86.206 & 0.431 \\
\hline 5 & 2.0 & 9.6 & 98.107 & 0.471 & 84.918 & 0.443 \\
\hline 6 & 2.0 & 9.3 & 98.330 & 0.404 & 86.846 & 0.375 \\
\hline 7 & 6.0 & 8.7 & 282.520 & 1.248 & 249.93 & 1.566 \\
\hline 1 & 0.5 & 9.6 & 25.858 & 0.096 & 23.158 & 0.145 \\
\hline 2 & 0.5 & 8.2 & 22.868 & 0.103 & 21.399 & 0.216 \\
\hline 3 & 0.5 & 10.1 & 22.423 & 0.203 & 20.813 & 0.187 \\
\hline 4 & 0.5 & 8.3 & 21.493 & 0.144 & 19.978 & 0.134 \\
\hline 5 & 0.5 & 9.5 & 21.451 & 0.173 & 17.883 & 0.155 \\
\hline 6 & 0.5 & 9.2 & 21.411 & 0.147 & 19.206 & 0.119 \\
\hline 7 & 0.5 & 8.6 & 19.947 & 0.063 & 17.682 & 0.180 \\
\hline
\end{tabular}

using reflectance-based or airborne sensor measurements, another important approach is to calibrate the OCI with respect to calibrated radiance data measured by other orbital sensors at similar times.

\subsection{Cross-platform in-flight calibration}

Cross-platform in-flight calibration of the OCI by using other calibrated ocean colour imagery to assess the top of the atmosphere radiance presents difficulties resulting from differences in spectral matching, ground spatial resolution, Sun-sensor geometry (Che et al. 1991), problems with radiometric compatibility and data set registration. These points are now investigated further.

\subsubsection{Spectral matching}

Table 5 shows the matching and partially overlapping spectral bands of several ocean colour sensors. The Ocean Color and Temperature Scanner (OCTS) on the ADEOS satellite is a product of the National Space Development Agency (NASDA) of Japan. It was launched in 1996, but failed to operate in February 1997. The Seaviewing Wide Field-of-view Sensor (SeaWiFS) on the SeaStar satellite is a product of the National Aeronautics and Space Administration (NASA) Office of Space and Science Applications and the Goddard Space Flight Center (GSFC) of the United States. SeaWiFS was successfully launched in 1997. The Moderate Resolution Imaging Spectroradiometer (MODIS) on the Earth Observing System's (EOS) AM-1 spacecraft, scheduled for launch in 1999, is also a NASA programme. The Coastal Zone Color Scanner (CZCS) on Nimbus-7, a pioneer of in-orbit ocean colour sensing, 
Table 5. Spectral matching among OCI, SeaWiFS, MODIS, OCTS and CZCS. Overlapping bands are listed in the same rows. The thick line blocks in one row indicate the matching bands. MODIS has 36 bands, only ocean colour bands are presented here. Wavelengths are in nanometres except where denoted.

\begin{tabular}{|c|c|c|c|c|}
\hline OCI & OCTS & SeaWiFS & MODIS & $\mathrm{CZCS}$ \\
\hline & $402-422$ & $402-422$ & $407.5-422.5$ & \\
\hline $433-453$ & $433-453$ & $433-453$ & $438-448$ & $433-453$ \\
\hline $480-500$ & $480-500$ & $480-500$ & $485-495$ & \\
\hline \multirow[t]{2}{*}{$500-520$} & $510-530$ & $500-520$ & & $510-530$ \\
\hline & & & $526-536$ & \\
\hline $545-565$ & $555-575$ & 545-565 & $550-560$ & $540-560$ \\
\hline \multirow[t]{3}{*}{$660-680$} & $655-675$ & $660-680$ & $662-672$ & $660-680$ \\
\hline & & & $676-686$ & 77080 \\
\hline & 745-785 & 745-785 & 745-755 & \\
\hline \multirow[t]{2}{*}{ 845-885 } & 845-885 & $845-885$ & $857.5-872.5$ & \\
\hline & $\begin{array}{l}3.55-3.88 \mu \mathrm{m} \\
8.25-8.8 \mu \mathrm{m} \\
10.3-11.4 \mu \mathrm{m} \\
11.4-12.5 \mu \mathrm{m}\end{array}$ & & & $10.5-12.5 \mu \mathrm{m}$ \\
\hline
\end{tabular}

ceased operating in 1986. As shown in table 5, three of the OCTS bands match OCI Bands 1, 2 and 6, and another three bands partially overlap OCI Bands 3, 4 and 5. SeaWiFS has six bands matching each OCI band. MODIS has five bands partially overlapping with Bands 1, 2, 4, 5 and 6 of the OCI. The CZCS has bands matching OCI Bands 1, 4 and 5. In spite of the partially overlapping bands, when taking uncertainties in physical implementation into consideration, no bands match each other perfectly. Table 2 showed that the bandwidths of the OCI are implemented slightly differently from their specified values. Therefore, to reduce spectral mismatch uncertainties, the selection of appropriate surface sites for cross-platform calibration should be considered. However, the good match between OCI and SeaWiFS bands should maximize the appropriate surface sites. In other words, most of the open sea under cross-observations of OCI and SeaWiFS should be appropriate candidates. Homogeneous ground sites with uniform reflectance are another choice, but in such cases the saturation conditions of the detectors must be considered. For sensors with partially overlapping bands, such as MODIS, the selection of surface sites for OCI cross-platform calibration is limited and spectral mismatch should be investigated carefully.

\subsubsection{Ground spatial resolution}

The resolutions at swath centre are approximately $800 \mathrm{~m} \times 800 \mathrm{~m}$ for a doublecell pixel of the OCI, $1100 \mathrm{~m} \times 1100 \mathrm{~m}$ for local area coverage (LAC) and $4500 \mathrm{~m} \times 4500 \mathrm{~m}$ for global area coverage $(\mathrm{GAC})$ of SeaWiFS, and $1000 \mathrm{~m} \times 1000 \mathrm{~m}$ 
for the ocean colour bands of MODIS. To account for differences in ground spatial resolution, multiple pixels of each instrument with the same ground coverage are compared in the cross-platform calibration. Assume that $I^{\mathrm{OCI}} J^{\mathrm{OCI}}$ pixels, i.e. $I$ pixels per line $\times J$ lines, at swath centre of OCI have the same ground coverage as $I^{\mathrm{v}} J^{\mathrm{v}}$ pixels of the cross-compared vicarious orbital sensor. The at-sensor radiance data corresponding to a double-cell OCI pixel can then be assessed by measuring a homogeneous surface site with uniform reflectance as follows:

$$
\bar{L}_{b p}^{v}(t)=\frac{1}{I^{\mathrm{OCI}} J^{\mathrm{OCl}}} \sum_{j=1}^{J^{\mathrm{v}}} \sum_{i=1}^{I^{\mathrm{v}}} \hat{L}_{b p_{i j}^{v}}^{v}(t)
$$

where the overbar indicates averaging. Atmosphere changes as a result of off-nadir observations of wide FOV instruments in different orbiting conditions make crossplatform comparisons very difficult. Pixel-by-pixel assessments of at-sensor radiance data by this method is also difficult. Consider decomposing the relative sensor gain as follows:

$$
\alpha_{b p}(t)=\alpha_{b}(t) \alpha_{p}(t)
$$

where $\alpha_{b}(t)$ and $\alpha_{p}(t)$ are respectively band-common and pixel-dependent portions of the relative sensor gain. Precise assessments of the pixel-dependent portions of the OCI gain can only be carried out by pre-flight calibration. Vicarious calibration for this purpose based on surface measurements is difficult due to its pushbroom scanning with wild FOV. Fortunately, except for changes in the responsivity of some CCD pixels and local contamination or damage of some optical component, the robust design of the OCI should ensure the pixel-dependent property to be maintained, and in most cases

$$
\alpha_{p}(t)=1.0
$$

is correct. Then, from equation (10) the band-common portion can be estimated from the following.

$$
\alpha_{b}(t)=\frac{\bar{L}_{b p \text { nadir }}^{\text {OCI }}(t)}{\bar{L}_{b p \text { nadir }}^{v}(t)}
$$

where

$$
\begin{gathered}
\bar{L}_{b p \text { nadir }}^{\mathrm{OCI}}(t)=\frac{1}{I^{\mathrm{OCI}} J^{\mathrm{OCI}}} \sum_{j=1}^{J^{\mathrm{OCI}} I_{i=1}^{\mathrm{OCI}}\left[L_{b p_{i j}}^{\mathrm{OCI}}(t)\right]} \\
L_{b p_{i j}}^{\mathrm{OCI}}(t)=\frac{\hat{C}_{b p_{i j} g}(t)-\hat{D}_{b p_{i j} g}(t)}{g F_{b p_{i j}}\left(t_{0}\right)}
\end{gathered}
$$

and $p_{i j}$ for $i=1, \ldots, I^{\mathrm{OCI}}$ and $j=1, \ldots, J^{\mathrm{OCI}}$ represents the pixels at swath centre. Referring to the architecture of the OCI, a shift in performance of the optics and electronic circuit units of a band will appear in the result of assessment by using (14).

\subsubsection{Sun-sensor geometry}

Sensors on different platforms will observe the target site under different illumination and viewing conditions. This introduces atmospheric changes and surface bi-directional reflectance effects. The OCI orbit is inclined at $35^{\circ}$ and has ground track crossing with sensors with Sun-synchronous orbits. To eliminate the effect of a different Sun-sensor geometry, an effective method is to perform cross-platform calibration by only using data obtained near in time over the cross points. 


\subsubsection{Radiometric compatibility}

The radiometric outputs of OCI, SeaWiFS, MODIS and OCTS were calibrated in the laboratory using known sources of spectral radiance such as integrating spheres and blackbody radiators. These sources refer to some primary standards maintained respectively by the United States and Japan. That means the same at-sensor radiance may result in differing spectral data from each of the ocean colour sensors. Compatibility of the output data of cross-compared sensors must therefore be investigated before launch. For this purpose, a radiometric comparison was carried out in February 1995 to calibrate and validate the OCTS integrating sphere with respect to the SeaWiFS and MODIS measurements (Johnson et al. 1997). The OCTS integrating sphere measured by the NEC Corporation in Yokohama, Japan yielded errors between $-2.7 \%$ and $3.9 \%$ (near zero on average) compared with the measurements of spheres for calibration of MODIS and SeaWiFS carried out in the United States. Although OCTS has failed to operate since February 1997, cross comparison with this sensor remains an important reference to the OCI because the same primary standard, integrating sphere and calibration method were applied to both OCTS and OCI (Narimatsu et al. 1997c). In turn, this means that the radiometric uncertainties of OCTS with respect to SeaWiFS and MODIS apply to OCI, and compatibility among the respective spectral data is ensured.

\subsubsection{Data set registration}

According to the method of cross-platform calibration described in \$5.1.2, the calibration requires data sets from around the cross point of the swath centre tracks of the OCI and the vicarious orbital sensor. Figure 6 shows the method of registration of the OCI against SeaWiFS, comprising image registration and data set registration.

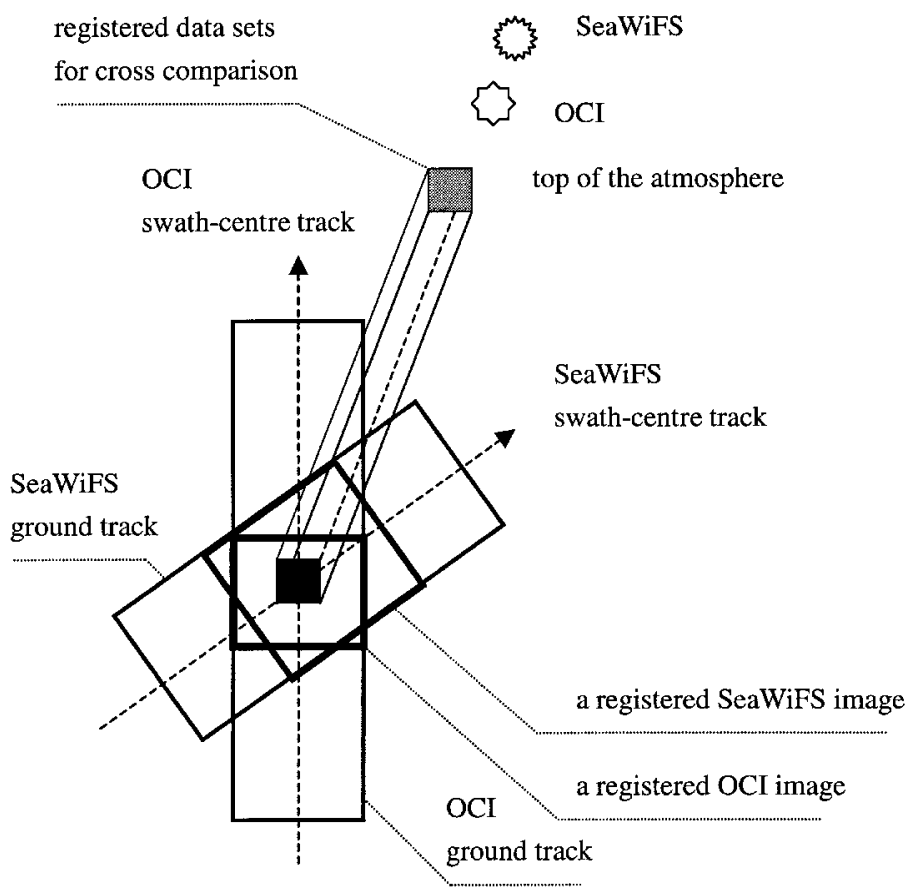

Figure 6. A schematic representation of image and data set registrations. 
Image registration is performed by commanding the two sensors to take images near in time (around $11 \mathrm{a} . \mathrm{m}$. for OCI and SeaWiFS) over some surface site where the swath centre tracks cross each other. Data set registration is extraction of the required data around the cross point of the swath centre tracks from each of the images. Data set registration can be achieved by image processing or by other computer-aided data handling techniques on the ground segment. As mentioned before the registered data sets from each of the images should have the same ground coverage over the cross point. If the surface site around the cross point is homogeneous and uniform in reflectance, the same ground coverage in area instead of perfect matching will be accurate enough for the calibration. Image registration requires the parameters of the orbits and pointing of the two sensors (satellites), so that the time and coordinates of the cross points can be computed in advance in order to set up the imaging commands. Alternatively, a particular surface site can be chosen for the cross-platform calibration, but the data will not be available until the two sensors fly over there near in time. However, if vicarious calibration based on measurements of surface reflectance is carried out within the same site, the calibration results can be double checked.

\subsection{Cross-band in-flight calibration}

Bands 4 and 7 of the OCI are observed through different telescopes but are spatially well co-registered and spectrally redundant. Thus they will be able to observe the same surface targets through the same atmosphere simultaneously. By comparing both data sets, a fault detection function can be developed to determine when to operate the on-duty band. The fault detection is designed to check the following parameters:

$$
\beta_{b 4,7 p}(t)=\frac{\left|\hat{L}_{b 4 p}(t)-\hat{L}_{b\urcorner p}(t)\right|}{\operatorname{Max}\left\{\hat{L}_{b 4 p}(t), \hat{L}_{b\urcorner p}(t)\right\}}
$$

where $\operatorname{Max}\left\{\hat{L}_{b 4 p}(t), \hat{L}_{b 7 p}(t)\right\} \neq 0$. A fault is detected if

$$
\beta_{b 4,7 p}(t) \geqslant \varepsilon
$$

where $\varepsilon, 0 \leqslant \varepsilon<1$ is a specified threshold. Detection of a fault will request a calibration to these two bands so that the abnormal band can be identified. When no fault is found, Band 4 can be calibrated with respect to Band 7 or vice versa. This may happen when one uses either NI-A or NI-B mode to collect data for the in-flight calibration, and invokes a CA (calibration) mode imaging to provide data for the calibration of Band 4 or 7. For the cross-band calibration, the offsets are calibrated as described before. However, since pixel-by-pixel cross-calibration is possible, the relative sensor gains can be assessed using equation (10) or, more specifically,

$$
\alpha_{b 4 p}(t)=\frac{\hat{C}_{b 4 p g}(t)-\hat{D}_{b 4 p g}(t)}{g F_{b 4 p}\left(t_{0}\right) \hat{L}_{b 7 p}^{\mathrm{v}}(t)} \quad \text { or } \quad \alpha_{b 7 p}(t)=\frac{\hat{C}_{b 7 p g}(t)-\hat{D}_{b 7 p g}(t)}{g F_{b 7 p}\left(t_{0}\right) \hat{L}_{b 4 p}^{\mathrm{v}}(t)}
$$

To account for the uncertainties, each relative sensor gain is assessed as the average of the results of (19) for more than one scanning line.

\section{Conclusions}

The OCI is a compact pushbroom scanning sensor without any moving or rotating components, so that high reliability in performance is expected. However, designed without any onboard calibrator, vicarious in-flight calibration is necessary 
to monitor short- and long-term variations in performance. Among the reflectanceand radiance-based vicarious calibration methods, cross-platform in-flight calibration using calibrated top of the atmosphere radiance data measured by a vicarious orbital sensor over the target site seems to be a convenient approach. Fortunately, the spectral bands of the OCI were selected to match or overlap those of some other on-duty ocean colour sensors. This undoubtedly motivates parameter adaptation to take advantage of the well calibrated radiance data obtained by these spaceborne sensors. To realize this consideration, this paper has presented the required radiometric model, the pre-flight parameters and a method of cross-platform in-flight calibration to adapt the parameters. Once the parameters are available, the output data of the OCI can be converted by using the model to obtain at-sensor radiance data. The proposed methods of modelling and data conversion have been implemented (the computer software is not shown here) and future work will compare the results obtained with other reflectance- or radiance-based methods.

\section{Acknowledgments}

This work is one of the projects of the OCI Science Team supported by the National Space Program Office of the National Science Council under NSC-86-NSPO-A-OCI-019-01-03 and NSC-87-NSPO-A-OCI-019-01-03. W. S. Lin would like to acknowledge the support from Mr Y. Narimatsu and the members of the NEC Space Systems Division in Yokohama, Japan. The authors also wish to acknowledge the support from the members of the OCI team of the National Space Program Office in Taiwan.

\section{References}

Che, N. B., Grant, B. G., Flittner, D. E., Slater, P. N., Biggar, S. F., Jackson, R. D., and Moran, M. S., 1991, Results of calibration of the NOAA-11 AVHRR made by reference to calibrated SPOT imagery at White Sands, N. M. Proceedings of SPIE: Calibration of Passive Remote Observing Optical and Microwave Instrumentation, 1493, 182-194.

Gordon, H. R., 1998, In-orbit calibration strategy for ocean color sensors. Remote Sensing and Environment, 63, 265-278.

Hooker, S. B., Esaias, W. E., Feldman, G. C., GregG, W. W., and Mcclain, C. R., 1992, SeaWiFS technical report series volume 1. An overview of SeaWiFS and Ocean Color. Technical Memorandom 104566, NASA, Greenbelt, MD, USA.

Johnson, B. C., Sakuma, F., Butler, J. J., Biggar, S. F., Cooper, J. W., Ishida, J., and Suzuki, K., 1997, Radiometric measurement comparison using the Ocean Color Temperature Scanner (OCTS) visible and near infrared integrating sphere.

Narimatsu, Y., 1997a, Ocean Color Imager Instrument: critical design review. Report OCI-BS-244-NEC, NEC Space Systems Division, Yokohama, Japan, pp. 8-22.

Narimatsu, Y., and Watanabe, F., 1997b, ROCSAT-1 Program: Ocean Color Imager FM delivery data package. Report OCI-BS-037-NEC, NEC Space Systems Division, Yokohama, Japan.

Narimatsu, Y., Watanabe, F., and Suzuki, K., 1997c, ROCSAT-1 Ocean Color Imager calibration report. Report OCI-BS-039-NEC, NEC Space Systems Division, Yokohama, Japan.

NSPO OCI Team, 1993, Ocean Color Imager for ROCSAT: feasibility study final report. Report MMS/OCI/NT/93-032, National Space Program Office, National Science Council, Taiwan, R.O.C.

Ono, A., Sakuma, F., Arai, K., Yamaguchi, Y., Fujisada, H., Slater, P. N., Thome, K. J., Palluconi, F. D., and Kieffer, H. H., 1996, Pre-flight and in-flight calibration plan for ASTER. Journal of Atmospheric and Oceanic Technology, 13, 321-335. 
Rao, C. R. N., and Chen, J., 1994, Post-launch calibration of the visible and near infrared channels of the Advanced Very High Resolution Radiometer on NOAA-7, -9 and -11 spacecraft. Report No. NESDID 78, National Oceanic and Atmospheric Administration, Washington, DC, USA.

Salomonson, V. V., Barnes, W. L., Maymon, P. W., Montgomery, H. E., and Ostrow, H., 1989, MODIS: advanced facility instrument for studies of the Earth as a system. IEEE Transactions on Geoscience and Remote Sensing, 27, 145-152.

Schowengerdt, R. A., 1997a, Remote Sensing: Models and Methods for Image Processing (New York: Academic), pp. 310-318.

Schowengerdt, R. A., 1997b, Remote Sensing: Models and Methods for Image Processing (New York: Academic), pp. 69-94.

Slater, P. N., Biggar, S. F., Holm, R. G., Jackson, R. D., Mao, Y., Moran, M. S., Palmer, J. M., and YuAN, B., 1987, Reflectance- and radiance-based methods for the in-flight absolute calibration of multi-spectral sensors. Remote Sensing and Environment, 22, 11-37. 